\title{
Unitals with many Baer secants through a fixed point
}

\author{
Sara Rottey* Geertrui Van de Voorde ${ }^{\dagger}$
}

June 28, 2021

\begin{abstract}
We show that a unital $U$ in $\operatorname{PG}\left(2, q^{2}\right)$ containing a point $P$, such that at least $q^{2}-\epsilon$ of the secant lines through $P$ intersect $U$ in a Baer subline, is an ovoidal Buekenhout-Metz unital (where $\epsilon \approx 2 q$ for $q$ even and $\epsilon \approx q^{3 / 2} / 2$ for $q$ odd).
\end{abstract}

\section{Introduction}

This paper studies unitals in the Desarguesian projective plane of square order $q^{2}, q=p^{h}$, $p$ prime, denoted by $\mathrm{PG}\left(2, q^{2}\right)$. A unital $U$ in $\mathrm{PG}\left(2, q^{2}\right)$ is a set of $q^{3}+1$ points of $\mathrm{PG}\left(2, q^{2}\right)$ such that each line contains exactly 1 or $q+1$ points of $U$.

An example of a unital in $\mathrm{PG}\left(2, q^{2}\right)$ is given by the set of absolute points of a unitary polarity, called a classical unital. In [6], Buekenhout constructed a class of unitals, called ovoidal Buekenhout-Metz unitals (see Subsection 2.3). Every known unital can be obtained by this construction.

Combining the results of [13] (for $q$ odd and $q>3$ ), and [7] (for $q>2$ even and $q=3$ ) the following characterisation of ovoidal Buekenhout-Metz unitals is obtained.

Theorem 1.1. [13, 7] Let $U$ be a unital in $\mathrm{PG}\left(2, q^{2}\right), q>2$, containing a point $P$ such that all secants through $P$ intersect $U$ in a Baer subline, then $U$ is an ovoidal BuekenhoutMetz unital with special point $P$.

Moreover, two related characterisations were found for classical unitals.

Theorem 1.2. 4] Let $U$ be an ovoidal Buekenhout-Metz unital in $\operatorname{PG}\left(2, q^{2}\right)$ with special point $P$. If $U$ contains a secant not through $P$ intersecting $U$ in a Baer subline, then $U$ is classical.

Theorem 1.3. [2] Let $U$ be a unital in $\mathrm{PG}\left(2, p^{2}\right), p$ prime, such that $p\left(p^{2}-2\right)$ secants intersect $U$ in a Baer subline, then $U$ is classical.

\footnotetext{
*VUB, Department of Mathematics, Pleinlaan 2, 1050 Brussel, Belgium. Email: srottey@vub.ac.be

${ }^{\dagger}$ This author is a postdoctoral fellow of the Research Foundation Flanders (FWO - Vlaanderen). UGent, Department of Mathematics, Krijgslaan 281 - S22, 9000 Gent, Belgium.

Email: gvdvoorde@cage.ugent.be
} 
Concerning these three results, in [10, Open problems 4] the following question was posed:

What is the minimum required number of secants being Baer sublines, to conclude that a unital is an ovoidal Beukenhout-Metz unital?

In this paper, we will improve the result of Theorem 1.1, and find a new upper bound for the minimum required number of Baer secants through a fixed point of the unital. It is worth noticing that our Main Theorem implies the result of [13] and [7] for $q \geq 16$.

Main Theorem. Suppose $q$ and $\epsilon$ satisfy the conditions of Table 1, Let $U$ be a unital in $\mathrm{PG}\left(2, q^{2}\right)$ containing a point $P$ such that at least $q^{2}-\epsilon$ of the secants through $P$ intersect $U$ in a Baer subline, then $U$ is an ovoidal Buekenhout-Metz unital with special point $P$.

\begin{tabular}{ll}
$\epsilon$ & Conditions \\
\hline$\epsilon \leq q-3$ & $q$ even, $q \geq 16$ \\
$\epsilon \leq 2 q-7$ & $q$ even, $q \geq 128$ \\
& \\
$\epsilon \leq \frac{\sqrt{q} q}{4}-\frac{39 q}{64}-O(\sqrt{q})+1$ & $q$ odd, $q \geq 17, q=p^{2 e}, e \geq 1$ \\
$\epsilon \leq \frac{\sqrt{q} q}{2}-2 q$ & $q$ odd, $q \geq 17, q=p^{2 e+1}, e \geq 1$ or $q$ prime \\
$\epsilon \leq \frac{\sqrt{q} q}{2}-\frac{67 q}{16}+\frac{5 \sqrt{q}}{4}-\frac{1}{12}$ & $q$ odd, $q \geq 17, q=p^{h}, p \geq 5$ \\
$\epsilon \leq \frac{\sqrt{q} q}{2}-\frac{35 q}{16}-O(\sqrt{q})+1$ & $q$ odd, $q \geq 23^{2}, q=p^{h}, h$ even for $p=3, q \neq 5^{5}, 3^{6}$ \\
\hline
\end{tabular}

Table 1: Conditions for the Main Theorem

\section{Preliminaries}

\subsection{Sublines and subplanes in the ABB-representation}

A Baer subline in $\mathrm{PG}\left(1, q^{2}\right)$ is a set of $q+1$ points whose coordinates, with respect to three well-chosen distinct points, are in $\mathbb{F}_{q}$. Similarly, a Baer subplane of $\mathrm{PG}\left(2, q^{2}\right)$ is a set of $q^{2}+q+1$ points, whose coordinates with respect to a well-chosen frame, are contained in $\mathbb{F}_{q}$. The $q^{2}+q+1$ points of a Baer subplane in $\operatorname{PG}\left(2, q^{2}\right)$, together with the lines they induce, form a projective plane of order $q$.

André [1] and Bruck and Bose [5] independently found a representation of translation planes of order $q^{n}$, with kernel containing $\mathbb{F}_{q}$, in the projective space $\operatorname{PG}(2 n, q)$. We refer to this as the André/Bruck-Bose-representation or the ABB-representation. In this paper, we restrict ourselves to the case $n=2$. 
Let $\mathcal{S}$ be a line spread in $\mathrm{PG}(3, q)$. Embed $\mathrm{PG}(3, q)$ as a hyperplane $H_{\infty}$ in $\mathrm{PG}(4, q)$. Consider the following incidence structure $A(\mathcal{S})$ with point set $P$ and line set $L$, where incidence is natural:

$P$ : the affine points, i.e. the points of $\mathrm{PG}(4, q) \backslash H_{\infty}$,

$L$ : the planes of $\mathrm{PG}(4, q)$ intersecting $H_{\infty}$ exactly in a line of $\mathcal{S}$.

In [5] the authors showed that $A(\mathcal{S})$ is an affine translation plane of order $q^{2}$, and conversely, every such translation plane can be constructed in this way. The plane $A(\mathcal{S})$ is a Desarguesian affine plane $\operatorname{AG}\left(2, q^{2}\right)$ if and only if the spread $\mathcal{S}$ is Desarguesian. The projective completion $\overline{A(\mathcal{S})}$ of the affine plane $A(\mathcal{S})$ can be found by adding $H_{\infty}$ as the line $\ell_{\infty}$ at infinity, where the lines of $\mathcal{S}$ correspond to the points of $\ell_{\infty}$. Clearly, the projective completion $\overline{A(\mathcal{S})}$ is a Desarguesian projective plane $\operatorname{PG}\left(2, q^{2}\right)$ if and only if the spread $\mathcal{S}$ is Desarguesian.

In this paper, we will fix a line $\ell_{\infty}$ at infinity of $\mathrm{PG}\left(2, q^{2}\right)$ and consider the ABBrepresentation with respect to this line. The hyperplane at infinity of $\mathrm{PG}(4, q)$, corresponding to $\ell_{\infty}$, will be denoted by $H_{\infty}$ and the Desarguesian spread defining $\operatorname{PG}\left(2, q^{2}\right)$ by $\mathcal{D}$.

We will call a Baer subline tangent (to $\ell_{\infty}$ ) if it has one point in common with $\ell_{\infty}$, and external if it has no such intersection point. In the ABB-representation, tangent sublines of $\mathrm{PG}\left(2, q^{2}\right)$ are in one-to-one correspondence with lines of $\mathrm{PG}(4, q)$ intersecting $H_{\infty}$ in exactly one point. An external subline corresponds to a non-degenerate conic of PG $(4, q)$, called a Baer conic, contained in a plane which meets $H_{\infty}$ in a spread line of $\mathcal{D}$, external to this conic. Note that, unless $q=2$, not every conic is a Baer conic. Moreover, since any two distinct Baer sublines have at most two points in common, we also have that any two distinct Baer conics share at most 2 points.

A Baer subplane will be called secant (to $\ell_{\infty}$ ) if it meets $\ell_{\infty}$ in $q+1$ points, and tangent if it meets $\ell_{\infty}$ in one point. In the ABB-representation, secant subplanes are in one-to-one correspondence with planes of $\mathrm{PG}(4, q)$ intersecting $H_{\infty}$ in a line not contained in $\mathcal{D}$. A tangent Baer subplane corresponds to the point set of $q+1$ disjoint lines, called generator lines, forming a ruled cubic surface, called a Baer ruled cubic. Such a Baer ruled cubic has a spread line $T \in \mathcal{D}$ as line directrix, where $T$ is the line corresponding to the intersection point of the tangent Baer subplane with $\ell_{\infty}$. As a base it has a Baer conic $C$ in a plane disjoint from $T$. For each point of $T$, there is a unique generator line on the Baer ruled cubic through this point and a point of $C$. A plane through a line of $\mathcal{D} \backslash\{T\}$ intersects the Baer ruled cubic in a point or a Baer conic. For more information on the ABB-representation of sublines and subplanes of $\mathrm{PG}\left(2, q^{2}\right)$, we refer to [3].

It is well-known that two distinct Baer sublines of $\mathrm{PG}\left(2, q^{2}\right)$, that have a common point, are contained in a unique Baer subplane. The following lemma, in terms of lines of $\mathrm{PG}(4, q)$ in the ABB-representation, can be deduced.

Lemma 2.1. Two lines of $\mathrm{PG}(4, q)$, not contained in a plane through a line of $\mathcal{D}$, intersecting $H_{\infty}$ in the same point, lie on a unique plane intersecting $H_{\infty}$ not in a line of $\mathcal{D}$, i.e. they define a unique secant subplane to $\ell_{\infty}$.

Two lines of $\mathrm{PG}(4, q)$, not in $H_{\infty}$, through different points $P_{1}, P_{2}$ of $H_{\infty}$, such that $P_{1} P_{2}$ is a spread line of $\mathcal{D}$, lie on a unique Baer ruled cubic, i.e. they define a unique tangent subplane to $\ell_{\infty}$. 


\subsection{Caps and ovoids in $\mathrm{PG}(3, q)$}

A $k$-cap in $\mathrm{PG}(3, q)$ is a set of $k$ points no three of which are collinear. A $k$-cap is called complete if it is not contained in a $(k+1)$-cap of $\mathrm{PG}(3, q)$. It is well-known that a cap of $\operatorname{PG}(3, q), q>2$, contains at most $q^{2}+1$ points; a $\left(q^{2}+1\right)$-cap is also called an ovoid.

We will need the following extendability results for caps in $\mathrm{PG}(3, q)$.

Theorem 2.2. A cap in $\mathrm{PG}(3, q)$, of size at least $q^{2}-\delta$, with $\delta$ and $q$ satisfying the conditions of Table 2, can be extended to an ovoid.

\begin{tabular}{lll}
\hline$\delta$ & Conditions & Reference \\
\hline$\delta \leq \frac{q}{2}+\frac{\sqrt{q}}{2}-1$ & $q$ even, $q>2$ & {$[12]$} \\
$\delta \leq q-4$ & $q$ even, $q \geq 8$ & {$[8]$} \\
$\delta \leq 2 q-8$ & $q$ even, $q \geq 128$ & \\
$\delta \leq \frac{\sqrt{q} q}{4}-\frac{39 q}{64}-O(\sqrt{q})$ & $q$ odd, $q \geq 17, q=p^{2 e}, e \geq 1$ \\
$\delta \leq \frac{p^{e+1} q}{4}-\frac{119 p q}{64}+O\left(p^{e+2}\right)$ & $q$ odd, $q \geq 17, q=p^{2 e+1}, e \geq 1$ \\
$\delta \leq \frac{359 q^{2}}{2700}+\frac{4 q}{135}-\frac{94}{27}$ & $q$ odd, $q \geq 17$ prime \\
$\delta \leq \frac{\sqrt{q} q}{2}-\frac{67 q}{16}+\frac{5 \sqrt{q}}{4}-\frac{13}{12}$ & $q$ odd, $q \geq 17, q=p^{h}, p \geq 5$ & $11]$ \\
$\delta \leq \frac{\sqrt{q} q}{2}-\frac{35 q}{16}-O(\sqrt{q})$ & $q$ odd, $q \geq 23^{2}, q=p^{h}$ \\
\hline
\end{tabular}

Table 2: Conditions for Theorem 2.2

Moreover, the following theorem shows that the ovoids obtained in the previous theorem are unique.

Theorem 2.3. [14, Theorem 2.2]

If $K$ is a $k$-cap in $\mathrm{PG}(n, q), n \geq 3, q$ even, having size $k>\left(q^{n-1}+\cdots+q+2\right) / 2$, then $K$ can be extended in a unique way to a complete cap.

If $K$ is a $k$-cap in $\mathrm{PG}(n, q), n \geq 3, q$ odd, of size $k>2\left(q^{n-1}+\cdots+q+2\right) / 3$, then $K$ can be extended in a unique way to a complete cap.

\subsection{Unitals in $\mathrm{PG}\left(2, q^{2}\right)$}

Recall that a unital in $\mathrm{PG}\left(2, q^{2}\right)$ is a set of $q^{3}+1$ points such that every line meets $U$ in 1 or $q+1$ points. It is easy to see that a point $P$ of $U$ lies on exactly one tangent line to 
$U$ and on $q^{2}$ lines meeting $U$ in $q+1$ points (including $P$ ). These last lines are called the $(q+1)$-secants, or short secants, to $U$. If a secant line meets a unital in a Baer subline, then we call this line a Baer secant.

A classical unital (or Hermitian curve) in $\mathrm{PG}\left(2, q^{2}\right)$ corresponds to the set of absolute points of a unitary polarity. Note that every unital in $\operatorname{PG}(2,4)$ is classical. In $\operatorname{PG}\left(2, q^{2}\right)$, $q>2$, there are examples of non-classical unitals.

An ovoidal Buekenhout-Metz unital in $\mathrm{PG}\left(2, q^{2}\right)$ arises from the following construction (see [6]). Consider the ABB-representation in $\operatorname{PG}(4, q)$ of $\operatorname{PG}\left(2, q^{2}\right)$ with respect to the line $\ell_{\infty}$, with line spread $\mathcal{D}$ of $H_{\infty}$ corresponding to the points of $\ell_{\infty}$. Let $\mathcal{O}$ be an ovoid in $\mathrm{PG}(4, q)$ intersecting $H_{\infty}$ in a unique point $A$, such that the tangent plane of $\mathcal{O}$ at $A$ does not contain the spread line $T \in \mathcal{D}$ incident with $A$. Let $V$ be a point on $T, V \neq A$. Consider the ovoidal cone with vertex $V$ and base $\mathcal{O}$, this point set forms a unital $U$ in $\mathrm{PG}\left(2, q^{2}\right)$. The line $\ell_{\infty}$ is the tangent line to $U$ at the point $P_{\infty}$ of $\ell_{\infty}$, where $P_{\infty}$ is the point corresponding to the spread line $T$. We will call $P_{\infty}$ the special point of the ovoidal Buekenhout-Metz unital $U$. Clearly, all secants to $U$ at $P_{\infty}$ are Baer secants.

All known unitals in $\mathrm{PG}\left(2, q^{2}\right)$, including the classical unital, arise as ovoidal BuekenhoutMetz unitals.

\section{Unitals with a point lying on many Baer secants}

In this section, we will prove our main theorem. We will need the following lemma which can be shown by a simple counting argument.

Lemma 3.1. [7, Theorem 2.1] A tangent Baer subplane meets a unital in $\mathrm{PG}\left(2, q^{2}\right)$ in at most $2 q+2$ points, a secant Baer subplane meets a unital in $\mathrm{PG}\left(2, q^{2}\right)$ in at most $2 q+1$ points.

Throughout this paper, we will use the following notations and conventions for a given unital $U$ of $\mathrm{PG}\left(2, q^{2}\right)$.

Let $U$ be a unital in $\mathrm{PG}\left(2, q^{2}\right)$ containing a point $P_{\infty}$ such that a set of at least $q^{2}-\epsilon$, $\epsilon \leq q^{2}$, of the $(q+1)$-secants through $P_{\infty}$ are Baer secants. Say $\ell_{\infty}$ is the tangent line of $U$ at $P_{\infty}$ and consider the ABB-representation of $\operatorname{PG}\left(2, q^{2}\right)$, where the points of $\ell_{\infty}$ correspond to the Desarguesian spread $\mathcal{D}$ of the hyperplane $H_{\infty}$ of $\mathrm{PG}(4, q)$. By abuse of notation, we will use the notation $U_{\text {aff }}$ for both the points of $U \backslash\left\{P_{\infty}\right\}$ in $\mathrm{PG}\left(2, q^{2}\right)$ and for the corresponding affine point set in $\mathrm{PG}(4, q)$.

Suppose $P_{\infty}$ corresponds to the spread line $T$ of $\mathcal{D}$. Let $\mathcal{L}$ be the set of $q^{2}-\epsilon$ lines in PG $(4, q)$ corresponding to Baer secants through $P_{\infty}$. Every line of $\mathcal{L}$ intersects $H_{\infty}$ in a point of $T$. Note that any plane intersecting $H_{\infty}$ in $T$ contains exactly $q$ points of $U_{\text {aff }}$.

Given a unital $U$ and its corresponding line set $\mathcal{L}$, we will consider a set $S(U)$ in the plane $\Pi=\operatorname{PG}(4, q) / T$, consisting of points with labels, induced by the lines of $\mathcal{L}$. This point set is defined as follows.

Definition. Consider the quotient space $\Pi=\operatorname{PG}(4, q) / T$, isomorphic to $\mathrm{PG}(2, q)$, and let $v_{1}, \ldots, v_{q+1}$ be the points of $T$. The points of $S(U)$ are the points of $\Pi$ corresponding to the planes through $T$ which contain a line of $\mathcal{L}$. We label a point $R$ of $S(U)$ with $v_{j}$, if the line of $\mathcal{L}$ in the plane $\langle T, R\rangle$ goes through $v_{j}$. 
Lemma 3.2. The set $S(U)$ is a point set in $\operatorname{AG}(2, q)$ such that each point has exactly one label. Moreover, $S(U)$ has the property that if a point $Q$ of $S(U)$ lies on a line of $\operatorname{AG}(2, q)$ containing two points of $S(U)$ with the same label $v$, then $Q$ also has label $v$.

Proof. First note that the points of $S(U)$ are contained in an affine plane of $\Pi=$ $\mathrm{PG}(4, q) / T$, since $H_{\infty} / T$ is a line in $\Pi$ and since no plane through $T$ in $H_{\infty}$ contains a line of $\mathcal{L}$. Each point of $S(U)$ has exactly one label, as a plane through $T$ contains at most one line of $\mathcal{L}$. If a line $m$ in $\Pi$ contains two points of $S(U)$ with the same label, say $v_{k}$, then the 3 -space $\langle T, m\rangle$ contains two lines $\ell_{1}, \ell_{2}$ of $\mathcal{L}$ through the point $v_{k}$. Suppose that there is a point of $S(U)$ on the line $m$ with label $v_{j}, j \neq k$. This implies that there is a line of $\mathcal{L}$, say $\ell_{3}$, through $v_{j}$, contained in $\langle T, m\rangle$. Thus, the line $\ell_{3}$ meets the plane $\left\langle\ell_{1}, \ell_{2}\right\rangle$ in an affine point, which means that the secant subplane defined by $\ell_{1}, \ell_{2}$ contains $2 q+2$ points, a contradiction by Lemma 3.1 .

Next, we show that the configuration of points of $S(U)$ must satisfy one of three conditions.

Lemma 3.3. Suppose $q>2$ and $k \in \mathbb{N}, k<\sqrt{q}-1$. Let $S$ be a set of $q^{2}-\epsilon, \epsilon \leq k q$, points in $\mathrm{AG}(2, q)$, and consider a set of labels $\mathcal{V}=\left\{v_{1}, \ldots, v_{q+1}\right\}$, such that each point of $S$ has exactly one label. Denote the subset of $S$ containing all points with label $v$ by $S_{v}$.

Suppose that the set $S$ has the property that if a point $Q$ of $S$ lies on a line of $\operatorname{AG}(2, q)$ containing two points of $S$ with the same label $v$, then $Q$ also has label $v$. Then the set $S$ satisfies one of the following.

(i) All points of $S$ have the same label.

(ii) There are 2 distinct labels $v_{1}$ and $v_{2}$ each occurring at least $q-k$ times as labels of points of $S$. For $i=1,2$, the points of $S_{v_{i}}$ lie on an affine line. These two affine lines go through a common affine point.

(iii) There is a subset $\mathcal{V}^{*} \subseteq \mathcal{V}$ of labels, each occurring at least twice, such that for every label $v \in \mathcal{V}^{*}$, the points of $S_{v}$ lie on an affine line. These affine lines are all parallel (i.e. their projective completions go through a common point $Q_{\infty}$ at infinity). The subset $S^{*} \subseteq S$, consisting of points with a label in $\mathcal{V}^{*}$, has size at least $q^{2}-\epsilon-\left(k^{2}+k\right)\left(k^{2}+k-1\right)-1$.

Proof. First, make the following two observations.

- Suppose that there is a label $v$ appearing $q+2$ times or more. Take a point $P \in S$, then at least one line through $P$ contains at least two points of $S$ with label $v$. Hence, the point $P$ also has label $v$, thus, all points of $S$ have label $v$. We find that $S$ has configuration $(i)$.

- Suppose that there is a label $v$, such that $q$ points of $S_{v}$ lie on a line $L$. If $S$ does not have configuration $(i)$, then one can check that no other point of $S$ has label $v$. Moreover, if another label appears at least two times, then the line spanned by the corresponding points must be parallel to $L$. Hence, any label appears at most $q$ times. There is a subset $\mathcal{V}^{*} \subseteq \mathcal{V}$ containing at least $q-k$ labels, such that every label 
appears at least twice; otherwise, there would be at most $(q-k-1) q+(k+2) 1=$ $q^{2}-k q-q+k+2<q^{2}-k q$ points in $S$. There are at most $k+1$ points having a label appearing only once. The subset $S^{*} \subseteq S$ of points having a label in $\mathcal{V}^{*}$ has size at least $q^{2}-\epsilon-k-1 \geq q^{2}-\epsilon-\left(k^{2}+k\right)\left(k^{2}+k-1\right)-1$. Hence, $S$ has the configuration described in (iii).

Now, consider a label $v$ occurring at least $q-k$ times. Suppose that there are three non-collinear points in $S_{v}$. Choose a point $P_{1} \in S_{v}$ and consider the set $Z$ of all lines containing $P_{1}$ and another point of $S_{v}$. Every line of $Z$ can only contain points with label $v$. Consider the set $Z^{\prime} \subseteq Z$ of all lines of $Z$ that contain at most $k$ points of $S$ different from $P_{1}$; suppose $\left|Z^{\prime}\right|=x$. Hence, the lines of $Z^{\prime}$ each contain at least $q-k-1$ affine points not in $S$. Since the lines of $Z^{\prime}$ contain at most all $k q$ points not in $S$, we see that

$$
x \leq \frac{k q}{q-k-1} .
$$

However, the upper bound for the number of points of $S_{v}$, different from $P_{1}$, covered by the lines of $Z^{\prime}$ is equal to $x k$. We see that

$$
x k \leq \frac{k^{2} q}{q-k-1}
$$

Moreover, when $k<\sqrt{q}-1$, we have

$$
\frac{k^{2} q}{q-k-1}<q-k-1
$$

As there are at least $q-k-1$ points in $S_{v}$, different from $P_{1}$, there exists a point $P_{2} \in S_{v}$ not on a line of $Z^{\prime}$. Hence, the line $P_{1} P_{2}$ contains at least $k+1$ points of $S$, different from $P_{1}$.

Consider a point $P_{3} \in S_{v}$, but not on $P_{1} P_{2}$. There are at least $k+2$ lines through $P_{3}$ and a point of $S \cap P_{1} P_{2}$ containing only points of $S$ with label $v$. These lines cover at least $1+(k+2)(q-1)-k q=2 q-k-1 \geq q+2$ points of $S$, when $k<\sqrt{q}-1$ and $q>2$. Since the label $v$ appears at least $q+2$ times, it follows that all points of $S$ have label $v$, hence, $S$ has configuration $(i)$.

We can now assume that if a label $v$ appears at least $q-k$ times, then the points of $S_{v}$ lie on a line. Moreover, since $q$ points with a fixed label on a line imply configuration (i) or (iii), we can pose that $\forall v \in \mathcal{V}:\left|S_{v}\right|<q$. We can count that there are at least two labels $v_{1}$ and $v_{2}$ each occurring at least $q-k$ times, since otherwise there would be at most $1(q-1)+q(q-k-1)=q^{2}-k q-1<q^{2}-k q$ points in $S$. Consider the lines $L_{1}$ and $L_{2}$ containing all points of $S_{v_{1}}$ and $S_{v_{2}}$ respectively.

If $L_{1}$ and $L_{2}$ intersect in an affine point $Q$, then $S$ has configuration $(i i)$.

Now, suppose $L_{1}$ and $L_{2}$ are parallel, i.e. their projective completions intersect in a point $Q_{\infty}$ at infinity. There are at least $q-k+1$ labels occurring at least twice, since otherwise there would be at most $(q-k)(q-1)+(k+1) 1=q^{2}-k q-q+2 k+1<q^{2}-k q$ points in $S$. A line spanned by two points with the same label (different from $v_{1}$ and $v_{2}$ ) must intersect both lines $L_{i}$ in a point not in $S$. However, the line $L_{i}, i=1,2$, contains at most $k$ affine points not in $S$. Hence, there are at most $k^{2}$ lines intersecting both lines $L_{i}$, 
$i=1,2$, not in $Q_{\infty}$ and not in a point of $S$. This means that, of all the labels appearing at least twice, there are at most $k^{2}$ labels such that two points with the same label do not necessarily span a line containing $Q_{\infty}$. Hence, there is a subset $\mathcal{V}^{*} \subseteq \mathcal{V}$ of at least $q-k^{2}-k+1$ labels occurring at least twice such that points with the same label do lie on a line containing $Q_{\infty}$.

It follows that there are at most $k^{2}+k-1$ affine lines through $Q_{\infty}$, such that the points of $S$ on such a line do not have the same label. However, there are at most $(q+1)-\left(q-k^{2}-k+1\right)=k^{2}+k$ labels that could occur this way. Hence, at most $\left(k^{2}+k-1\right)\left(k^{2}+k\right)$ points of $S$ have the property that a line spanned by two points with the same label does not necessarily contain $Q_{\infty}$. It follows that there is a subset $S^{*} \subseteq S$ of at least $q^{2}-\epsilon-\left(k^{2}+k\right)\left(k^{2}+k-1\right)>q^{2}-\epsilon-\left(k^{2}+k\right)\left(k^{2}+k-1\right)-1$ points, having the property that a line spanned by two points with the same label does contain $Q_{\infty}$, i.e. they have a label in $\mathcal{V}^{*}$. This means that $S$ has configuration (iii).

The following three lemmas will show that the affine point set $S(U)$, defined by the unital $U$, must satisfy the first configuration of Lemma 3.3.

The subset of $S(U)$ containing all points with label $v_{i}$, will be denoted by $S_{v_{i}}(U)$.

Lemma 3.4. Suppose $q>2$ and $k \in \mathbb{N}, k<\sqrt{q}-1$. Let $U$ be a unital containing a point $P_{\infty}$ such that $q^{2}-\epsilon, \epsilon \leq k q$, of the $(q+1)$-secants through $P_{\infty}$ are Baer secants. The corresponding point set $S(U)$ cannot have the form (ii) of Lemma 3.3.

Proof. Suppose that $S(U)$ is of the form $(i i)$ of Lemma 3.3. There are two distinct labels, say $v_{1}$ and $v_{2}$, occurring at least $q-k$ times, such that for $i=1,2$, the points of $S_{v_{i}}(U)$ lie on an affine line $L_{i}$. The affine lines $L_{1}$ and $L_{2}$ intersect in an affine point $A$.

Let $T$ be the spread line corresponding to $P_{\infty}$. A line of $\mathcal{L}$ through $v_{1}$ induces a point of $L_{1}$ in the quotient space $\mathrm{PG}(4, q) / T$. Hence, all the lines of $\mathcal{L}$ containing $v_{1}$ are contained in the three-space $\Sigma_{1}=\left\langle T, L_{1}\right\rangle$. Similarly, the lines of $\mathcal{L}$ containing $v_{2}$ are contained in the three-space $\Sigma_{2}=\left\langle T, L_{2}\right\rangle$. Let $\alpha$ be the plane $\langle T, A\rangle$, then clearly $\alpha$ is the intersection $\Sigma_{1} \cap \Sigma_{2}$. Moreover, as the plane $\alpha$ is not contained in $H_{\infty}$, there are $q$ points of $U_{\text {aff }}$ contained in $\alpha$.

There are at most $k+1$ lines, say $n_{1}, \ldots, n_{k+1}$, of $\alpha$ through $v_{1}$ which do not occur as the intersection $\left\langle\ell_{i}, \ell_{j}\right\rangle \cap \alpha$, where $\ell_{i}, \ell_{j}$ are lines of $\mathcal{L}$ through $v_{1}$ in the three-space $\Sigma_{1}$. Similarly, there are at most $k+1$ lines $n_{1}^{\prime}, \ldots, n_{k+1}^{\prime}$ of $\alpha$ through $v_{2}$ which do not occur as the intersection $\left\langle\ell_{i}, \ell_{j}\right\rangle \cap \alpha$, where $\ell_{i}, \ell_{j}$ are lines of $\mathcal{L}$ through $v_{2}$ in the three-space $\Sigma_{2}$.

Suppose that a point of $U$ in $\alpha$ lies on a plane $\left\langle\ell_{i}, \ell_{j}\right\rangle$, where $\ell_{i}, \ell_{j}$ are lines of $\mathcal{L}$ through the same point of $T$, then the secant subplane defined by $\ell_{i}, \ell_{j}$ contains $2 q+2$ points of $U$, a contradiction by Lemma 3.1. This implies that each of the $q$ points of $U$ in $\alpha$ necessarily lies on one of the lines $n_{1}, \ldots, n_{k+1}$ and on one of the lines $n_{1}^{\prime}, \ldots n_{k+1}^{\prime}$. However, there are only $(k+1)^{2}$ such points and $q>(k+1)^{2}$, a contradiction.

Consider a Baer subplane $\pi$ of $\mathrm{PG}\left(2, q^{2}\right)$ containing the point $P_{\infty}$. It is clear that $\pi / P_{\infty}$ defines a Baer subline in the quotient space $\mathrm{PG}\left(2, q^{2}\right) / P_{\infty}$. This can be translated to the ABB-representation in the following way. Recall that a Baer subplane $\pi$, tangent to $\ell_{\infty}$ at $P_{\infty}$, corresponds to a Baer ruled cubic $\mathcal{B}$ with line directrix $T$. We see that $\mathcal{B} / T$ defines a Baer conic in the quotient space $\mathrm{PG}(4, q) / T$. 
Lemma 3.5. Suppose $q \geq 16$ and $k \in \mathbb{N}, k \leq \sqrt{q} / 2-2$. Let $U$ be a unital containing a point $P_{\infty}$ such that $q^{2}-\epsilon, \epsilon \leq k q$, of the $(q+1)$-secants through $P_{\infty}$ are Baer secants. Suppose $S(U)$ is as described in Lemma 3.3 case (iii), with subset $S^{*}(U) \subseteq S(U)$. Then there exists a Baer ruled cubic $\mathcal{B}$ in $\mathrm{PG}(4, q)$, containing two lines of $\mathcal{L}=\left\{\ell_{1}, \ldots, \ell_{q^{2}-\epsilon}\right\}$, such that the corresponding Baer conic in $\mathrm{PG}(4, q) / T$ contains at least $\left\lfloor\frac{q+7}{2}\right\rfloor$ points of $S^{*}(U)$.

Proof. Consider $S(U)$ as described in Lemma 3.3 case (iii), with point $Q_{\infty}$ at infinity. There is a subset $S^{*}(U) \subseteq S(U)$ of at least $q^{2}-k q-\left(k^{2}+k\right)\left(k^{2}+k-1\right)-1$ points of $S(U)$, such that points of $S^{*}(U)$ with the same label lie on an affine line containing the point $Q_{\infty}$.

Choose a point $R \in S^{*}(U)$ having label $v$, this label $v$ occurs at most $q$ times. Hence, there are at least

$$
q^{2}-(k+1) q-\left(k^{2}+k\right)\left(k^{2}+k-1\right)-1
$$

points of $S^{*}(U)$, not with label $v$. We will call these points good points. The affine points which are not good, are called bad points.

Consider the line $\ell \in \mathcal{L}$ defined by $R$. We want to find a Baer ruled cubic, containing $\ell$, such that the corresponding Baer conic in $\mathrm{PG}(4, q) / T$ contains at least $\left\lfloor\frac{q+7}{2}\right\rfloor$ points of $S^{*}(U)$. Since such a conic always contains $R \in S^{*}(U)$, we want to find a conic with at least $\left\lfloor\frac{q+5}{2}\right\rfloor$ good points and at most $\left\lceil\frac{q-3}{2}\right\rceil$ bad points (one of which is $R$ ).

Consider a good point $R_{1}$ and its corresponding line $\ell_{1} \in \mathcal{L}$. As all good points have a label different from $v$, the points $R_{1}$ and $R$ have a different label. Hence, the lines $\ell$ and $\ell_{1}$ intersect $T$ in a distinct point, so they are contained in a unique Baer ruled cubic (by Lemma 2.1). Consider the corresponding Baer conic $C_{1}$ in $\operatorname{PG}(4, q) / T$. If the conic $C_{1}$ contains at least $\left\lfloor\frac{q+5}{2}\right\rfloor$ good points, the result follows. Now, suppose that $C_{1}$ contains at most $\left\lfloor\frac{q+3}{2}\right\rfloor$ good points. Then there are at least $q^{2}-(k+1) q-\left(k^{2}+k\right)\left(k^{2}+k-1\right)-1-\frac{q+3}{2}$ good points that do not belong to $C_{1}$. Since $q \geq 4(k+1)^{2}$, this number is greater than zero.

Hence, we can find a good point $R_{2}$ that does not lie on $C_{1}$. The point $R_{2}$ defines a line $\ell_{2}$ of $\mathcal{L}$. Again, we know that the lines $\ell$ and $\ell_{2}$ intersect $T$ in a different point. Take the Baer ruled cubic defined by $\ell$ and $\ell_{2}$, and consider the corresponding Baer conic $C_{2}$ in $\mathrm{PG}(4, q) / T$. Recall that two distinct Baer conics intersect in at most two points, hence $C_{2}$ meets $C_{1}$ in $R$ and in at most one other point. If the conic $C_{2}$ contains at least $\left\lfloor\frac{q+5}{2}\right\rfloor$ good points, the result follows. So, suppose that at most $\left\lfloor\frac{q+3}{2}\right\rfloor$ points of $C_{2}$ are good points.

Since $q^{2}-(k+1) q-\left(k^{2}+k\right)\left(k^{2}+k-1\right)-1-2 \frac{q+3}{2}>0$, we can find a good point $R_{3}$, not contained in $C_{1} \cup C_{2}$. Applying the same reasoning to $R_{3}$, we find a new Baer ruled cubic containing $\ell$. The corresponding Baer conic $C_{3}$ contains $R$ and $R_{3}$, and is different from both $C_{1}$ and $C_{2}$. Thus, $C_{3}$ meets both in at most 1 point different from $R$.

Continuing this reasoning, suppose we have $m=2 k^{2}+4$ Baer conics $C_{1}, \ldots, C_{m}$ through $R$, each containing at most $\left\lfloor\frac{q+3}{2}\right\rfloor \operatorname{good}$ points. Hence, there are still at least

$$
q^{2}-(k+1) q-\left(k^{2}+k\right)\left(k^{2}+k-1\right)-1-m \frac{q+3}{2}
$$

good points not contained in one of the conics $C_{i}, i=1, \ldots, m$. We obtain the parabola

$$
q^{2}-\left(k^{2}+k+3\right) q-\left(k^{4}+2 k^{3}+3 k^{2}-k+7\right)
$$


with largest zero point equal to

$$
q=\frac{\left(k^{2}+k+3\right)+\sqrt{\left(k^{2}+k+3\right)^{2}+4\left(k^{4}+2 k^{3}+3 k^{2}-k+7\right)}}{2} .
$$

Since

$$
q \geq 4(k+2)^{2}>\frac{\left(k^{2}+k+3\right)+\sqrt{\left(k^{2}+k+3\right)^{2}+4\left(k^{4}+2 k^{3}+3 k^{2}-k+7\right)}}{2},
$$

there is at least one good point not on $C_{1} \cup \ldots \cup C_{m}$, say $R_{m+1}$. Consider the line $\ell_{m+1} \in \mathcal{L}$ corresponding to $R_{m+1}$. The Baer ruled cubic $\mathcal{B}$ defined by $\ell$ and $\ell_{m+1}$ induces a Baer conic $C_{m+1}$ in $\mathrm{PG}(4, q) / T$.

There are at most $(k+1) q+\left(k^{2}+k\right)\left(k^{2}+k-1\right)+1$ bad points contained in $\mathrm{PG}(4, q) / T$. Each conic $C_{i}, i=1, \ldots, m$, contains at most $\left\lfloor\frac{q+3}{2}\right\rfloor \operatorname{good}$ points, hence at least $\left\lceil\frac{q-1}{2}\right\rceil$ bad points, one of which is $R$. Since two conics have at most one bad point in common different from $R$, the conics $C_{1}, \ldots, C_{m}$ cover at least $1+m\left\lceil\frac{q-3}{2}\right\rceil-\frac{m(m-1)}{2}$ bad points. The conic $C_{m+1}$ can intersect each conic $C_{i}, i=1, \ldots, m$, in at most one bad point. Hence, there are at most

$$
\begin{gathered}
1+m+\left[(k+1) q+\left(k^{2}+k\right)\left(k^{2}+k-1\right)+1\right]-\left[1+m \frac{q-3}{2}-\frac{m(m-1)}{2}\right] \\
=1+m+(k+1) q+\left(k^{2}+k\right)\left(k^{2}+k-1\right)-m \frac{q-3}{2}+\frac{m(m-1)}{2}
\end{gathered}
$$

bad points contained in $C_{m+1}$. To check that this number is strictly smaller than $\frac{q-1}{2}$, we find the inequality

$$
(-m+2 k+1) q+2\left(k^{2}+k\right)\left(k^{2}+k-1\right)+m^{2}+4 m+3<0 .
$$

This is equivalent to

$$
q>\frac{2\left(k^{2}+k\right)\left(k^{2}+k-1\right)+m^{2}+4 m+3}{m-2 k-1},
$$

which is valid when $q \geq 16$, since

$$
q \geq 4(k+2)^{2}>\frac{2\left(k^{2}+k\right)\left(k^{2}+k-1\right)+m^{2}+4 m+3}{m-2 k-1} .
$$

This means that the Baer ruled cubic $\mathcal{B}$ has at most $\left\lceil\frac{q-3}{2}\right\rceil$ bad points, hence at least $\left\lfloor\frac{q+5}{2}\right\rfloor$ good points. It follows that $\mathcal{B}$ contains at least $\left\lfloor\frac{q+7}{2}\right\rfloor$ points of $S^{*}(U)$ and thus satisfies the conditions of the statement.

Lemma 3.6. Suppose $q \geq 16$ and $k \in \mathbb{N}, k \leq \sqrt{q} / 2-2$. Let $U$ be a unital containing a point $P_{\infty}$ such that $q^{2}-\epsilon, \epsilon \leq k q$, of the $(q+1)$-secants through $P_{\infty}$ are Baer secants. The corresponding affine point set $S(U)$ cannot have the form (iii) of Lemma 3.3 . 
Proof. Suppose that the set $S(U)$ has the form (iii) of Lemma 3.3 with point $Q_{\infty}$ at infinity. Let $\ell_{1}$ and $\ell_{2}$ be the lines of $\mathcal{L}$ defining the Baer ruled cubic $\mathcal{B}$ of Lemma 3.5. A tangent subplane contains (at most) $2 q+2$ points of $U$, hence $\mathcal{B}$ contains (at most) one point of $U_{\text {aff }}$ not on $\ell_{1}$ and $\ell_{2}$. Let $\mu$ be a plane (necessarily skew from $T$ ) containing a Baer conic $C$ contained in $\mathcal{B}$. We can identify $\mathrm{PG}(4, q) / T$ with $\mu$, and so the intersection points of $U \cap \mathcal{B}$ define the points $R_{1}, R_{2}$ in $C$ (corresponding to $\ell_{1}$ and $\ell_{2}$ respectively) and at most one extra point $R$ in $C$.

By Lemma 3.5, there are at least $\left\lfloor\frac{q+7}{2}\right\rfloor$ points of the Baer conic $C$ contained in $S^{*}(U)$, that is, two points of $S^{*}(U)$ with the same label lie on a line containing $Q_{\infty}$. Hence, we find at least two lines $L_{A}$ and $L_{B}$ through $Q_{\infty}$, each intersecting $C$ in two points with the same label. At most one of these lines, say $L_{B}$, contains the point $R$. Hence, $L_{A}$ intersects $C \backslash\{R\}$ in two points $Q_{1}, Q_{2}$, having the same label $v$. The points $Q_{1}$ and $Q_{2}$ are each contained in a generator line of the Baer ruled cubic, say $n_{1}$ and $n_{2}$. Since $Q_{1}$ and $Q_{2}$ are different from $R$, for $i=1,2$, the line $n_{i}$ either has no affine intersection point with the lines of $\mathcal{L}$ or is equal to $\ell_{1}$ or $\ell_{2}$.

Both points $Q_{i}, i=1,2$, have label $v$, hence, the planes $\left\langle T, n_{i}\right\rangle, i=1,2$, each contain a line of $\mathcal{L}$ through $v$, say $\ell_{k_{1}}$ and $\ell_{k_{2}}$ respectively. Since the line $n_{i}$ is either equal to $\ell_{k_{i}}$ or does not have an affine intersection point with $\ell_{k_{i}}$, both lines $n_{i}, i=1,2$, have to meet $T$ in $v$. This implies that we find two generator lines of the same Baer ruled cubic having a point in common, a contradiction by the definition of a ruled cubic surface, which concludes the proof.

As a combination of previous lemma's, we have found that $S(U)$ must satisfy configuration $(i)$ of Lemma 3.3. We will show that in this case, the points of $U$ on the $q^{2}-\epsilon$ Baer secants are contained in a unique unital, namely an ovoidal Buekenhout-Metz unital. This leads to the conclusion that $U$ is an ovoidal Buekenhout-Metz unital.

First, we prove that $q^{2}-\epsilon$ Baer secants of an ovoidal Buekenhout-Metz unital are never contained in any other unital. We need the definition of an O'Nan configuration, this is a collection of four distinct lines meeting in six distinct points, as illustrated in the following picture.

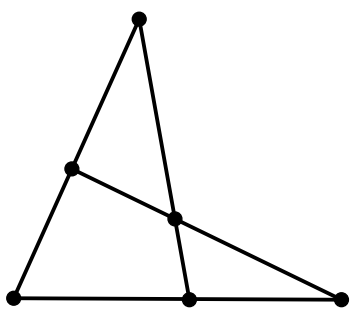

It is known that an ovoidal Buekenhout-Metz unital contains no O'Nan configurations through its special point. A simple proof of this can be found in the proof of [3, Lemma $7.42]$.

We will call a line of $\operatorname{PG}\left(2, q^{2}\right)$ which is secant to a unital $U^{\prime}$, a $U^{\prime}$-secant.

Lemma 3.7. Consider an ovoidal Buekenhout-Metz unital $U^{\prime}$ of $\operatorname{PG}\left(2, q^{2}\right)$ with special point $P_{\infty}$ and consider a set $\left\{L_{1}, \ldots, L_{\epsilon}\right\}$ of $U^{\prime}$-secants through $P_{\infty}$. Consider a unital $U$ of $\mathrm{PG}\left(2, q^{2}\right)$ containing $P_{\infty}$ and all points of $U^{\prime}$ that do not lie on one of the $\epsilon$ secant lines $L_{i}$. If $\epsilon \leq \frac{(q-1) q}{2}$, then $U$ and $U^{\prime}$ coincide. 
Proof. We will show that the result holds when $\epsilon=\frac{(q-1) q}{2}$, then the result easily follows for all $\epsilon \leq \frac{(q-1) q}{2}$.

Consider the set $U_{0}$ consisting of all points contained in $U^{\prime}$, but not on one of the $U^{\prime}$-secants $L_{i}, i=1, \ldots, \epsilon$. By assumption all these points are contained in $U \cap U^{\prime}$. Recall that for every unital $\widetilde{U}$, a point of $\widetilde{U}$ lies on $q^{2} \widetilde{U}$-secants and a point not on $\widetilde{U}$ lies on only $q^{2}-q \widetilde{U}$-secants. This means, if a point $Q$ lies on strictly more than $q^{2}-q$ lines intersecting $U_{0}$ in at least two points, then $Q$ is contained in any unital containing all points of $U_{0}$. Hence, in that case, $Q$ is contained in $U \cap U^{\prime}$.

Consider a point $R \in U^{\prime} \backslash U_{0}$ and say $L_{1}=P_{\infty} R$. We will prove that there are at most $q-2 U^{\prime}$-secants $M_{j}$, containing $R$ but different from $L_{1}$, having at most 1 point in common with $U_{0}$. If that is the case, then there are at least $q^{2}-q+1 U^{\prime}$-secants through $R$ containing at least two points of $U_{0}$, and hence, the point $R$ is contained in $U \cap U^{\prime}$.

Consider a $U^{\prime}$-secant $M_{1}$, different from $L_{1}$, containing $R$ and (at most) 1 point of $U_{0}$. This line intersects at least $q-1 U^{\prime}$-secants $L_{i}$, different from $L_{1}$, in a point of $U^{\prime}$, say $L_{2}, \ldots, L_{q}$.

Take a $U^{\prime}$-secant $M_{2}$ through $R$, different from $L_{1}$ and $M_{1}$, containing at most 1 point of $U_{0}$. Since $U^{\prime}$ contains no O'Nan configurations through the point $P_{\infty}$, there is at most one $U^{\prime}$-secant $L_{i}, i \neq 1$, containing $P_{\infty}$, such that the points $L_{i} \cap M_{1}$ and $L_{i} \cap M_{2}$ are both points of $U^{\prime}$. Hence, $M_{2}$ intersects at least $q-2$ new $U^{\prime}$-secants $L_{i}$ (i.e. different from $\left.L_{1}, \ldots, L_{q}\right)$ in a point of $U^{\prime}$, say $L_{q+1}, \ldots, L_{2 q-2}$.

Consider a third $U^{\prime}$-secant $M_{3}$ through $R$, different from $L_{1}, M_{1}, M_{2}$. With the same reasoning as above, $M_{3}$ intersects at least $q-3 U^{\prime}$-secants $L_{i}$ (different from $L_{1}, \ldots, L_{2 q-2}$ ) in a point of $U^{\prime}$, say $L_{2 q-1}, \ldots, L_{3 q-5}$.

If there are at most $q-2 U^{\prime}$-secants $M_{j}$, containing $R$ and having 0 or 1 points in common with $U_{0}$, the result follows. Otherwise, by continuing this process, the $U^{\prime}-$ secant $M_{q-1}$ intersects at least $q-(q-1)=1 U^{\prime}$-secant $L_{i}$, different from the previously enumerated lines $L_{1}, \ldots, L_{m}$. We have found $m+1$ distinct $U^{\prime}$-secants $L_{j}$ where

$$
m+1=1+(q-1)+(q-2)+\ldots+(q-(q-2))+1=\frac{q(q-1)}{2}+1 .
$$

This is in contradiction with the restriction on the number of $U^{\prime}$-secants $L_{j}$, since

$$
\frac{q(q-1)}{2}+1>\frac{q(q-1)}{2}=\epsilon
$$

We have proved that there are at most $q-2 U^{\prime}$-secants through $R$ containing 0 or 1 points of $U_{0}$. Hence, the point $R$ is contained in $U \cap U^{\prime}$. It follows that all points $R \in U^{\prime}$ are contained in $U \cap U^{\prime}$, which proves the result.

Lemma 3.8. Suppose $q$ and $\delta$ satisfy the conditions of Table Q Consider a unital $U$ containing a point $P_{\infty}$ such that at least $q^{2}-\delta-1$ of the $(q+1)$-secants through $P_{\infty}$ are Baer secants. If $S(U)$ satisfies configuration $(i)$ of Lemma 3.3, then $U$ is an ovoidal Buekenhout-Metz unital with special point $P_{\infty}$.

Proof. If the set $S(U)$ satisfies configuration $(i)$ of Lemma 3.3, then all points of $S(U)$ have the same label. This implies that all $q^{2}-\delta-1$ lines of $\mathcal{L}$ go through a common point, say $v$ of the line $T$. By Lemma 2.1, two lines $\ell_{i}$ and $\ell_{j}$ of $\mathcal{L}$ define a unique secant 
subplane. By Lemma 3.1, such a subplane has no affine intersection with any other line of $\mathcal{L}$. This means that in the 3 -dimensional quotient space $\operatorname{PG}(4, q) / v$, the lines of $\mathcal{L}$ define a set $K$ of $q^{2}-\delta-1$ points forming a cap. As a plane through $T$ contains at most one line of $\mathcal{L}$, the line $T$ defines a point in this quotient space, which extends the cap $K$ to a cap $K^{\prime}$ of size $q^{2}-\delta$. By Theorems 2.2 and 2.3, the cap $K$ can be extended to a unique ovoid $\mathcal{O}$. The cone with vertex $v$ and base $\mathcal{O}$ defines an ovoidal Buekenhout-Metz unital $U^{\prime}$ which has $q^{2}-\delta-1$ secant lines in common with $U$. Since $\delta+1 \leq \frac{(q-1) q}{2}$, by Lemma 3.7, $U$ is an ovoidal Buekenhout-Metz unital.

Main Theorem. Suppose that $q$ and $\epsilon$ satisfy the conditions of Table 1 . Let $U$ be a unital containing a point $P_{\infty}$ such that at least $q^{2}-\epsilon$ of the $(q+1)$-secants through $P_{\infty}$ are Baer secants, then $U$ is an ovoidal Buekenhout-Metz unital with special point $P_{\infty}$.

Proof. When $q$ and $\epsilon$ satisfy the conditions of Table 1, we have $q \geq 16$ and $\epsilon \leq \min (\delta+$ $1, \sqrt{q} q / 2-2 q)$ with $q$ and $\delta$ satisfying the conditions of Table 2 ,

Consider the set $S(U)$ defined by the Baer secants to $U$ at $P_{\infty}$. By Lemma 3.2, this set satisfies the conditions of Lemma 3.3. Hence, since $q>2$ and $\epsilon<(\sqrt{q}-1) q$, the set $S(U)$ has one of the three configurations of Lemma 3.3. By Lemma 3.4 ( $q>2$ and $\epsilon<(\sqrt{q}-1) q)$ and Lemma 3.6 ( $(q \geq 16$ and $\epsilon \leq \sqrt{q} q / 2-2 q)$, only the first configuration is possible. Since $\epsilon \leq \delta+1$, by Lemma 3.8 , $U$ is an ovoidal Buekenhout-Metz unital.

Combining the Main Theorem with Theorem [1.2, we obtain the following corollary.

Corollary 3.9. Suppose that $q$ and $\epsilon$ satisfy the conditions of Table 1. Let $U$ be a unital in $\mathrm{PG}\left(2, q^{2}\right)$. If there is a point $P_{\infty}$ in $U$ that lies on at least $q^{2}-\epsilon$ Baer secants, and there exists a Baer secant of $U$ not through $P_{\infty}$, then $U$ is a classical unital.

\section{References}

[1] J. André. Über nicht-Dessarguessche Ebenen mit transitiver Translationsgruppe. Math Z. 60 (1954), 156-186.

[2] S. Ball, A. Blokhuis and C.M. O'Keefe. On unitals with many Baer sublines. Des. Codes Cryptogr. 17 (1999), 237-252.

[3] S.G. Barwick and G. Ebert. Unitals in projective planes. Springer Monographs in Mathematics. Springer, New York, 2008.

[4] S.G. Barwick and C.T. Quinn. Generalising a characterisation of Hermitian curves. J. Geom. 70 (1-2) (2001), 1-7.

[5] R.H. Bruck and R.C. Bose. The construction of translation planes from projective spaces. J. Algebra 1 (1964), 85-102.

[6] E. Buekenhout. Existence of unitals in finite translation planes of order $q^{2}$ with a kernel of order q. Geom. Dedicata 5 (1976), 189-194.

[7] L.R. Casse, C.M. O'Keefe and T. Penttila. Characterizations of Buekenhout-Metz unitals. Geom. Dedicata 59 (1) (1996), 29-42. 
[8] J. Cao and L. Ou. Caps in $\operatorname{PG}(n, q)$ with $q$ even and $n \geq 3$. Discrete Math. 326 (2014), 61-65.

[9] J.M. Chao. On the size of a cap in $\operatorname{PG}(n, q)$ with $q$ even and $n=3$. Geom. Dedicata 74 (1999), 91-94.

[10] F. De Clerck and N. Durante. Constructions and characterizations of classical sets in PG(n,q). In Current Research Topics in Galois Geometry. J. De Beule and L. Storme (Eds.). Nova Science, New York, 2012, Ch. 1, 1-33.

[11] J. Hirschfeld and L. Storme. The packing problem in statistics, coding theory and finite projective geometries, update 2001. In Finite geometries, Proceedings of the Fourth Isle of Thorns Conference. A. Blokhuis, J.W.P. Hirschfeld, D. Jungnickel and J.A. Thas (Eds.). Dev. Math 3, Kluwer Acad. Publ., Dordrecht (2001), 201-246.

[12] J.W.P. Hirschfeld and J.A. Thas. Linear independence in finite spaces. Geom. Dedicata 23 (1987), 15-31.

[13] C.T. Quinn and R. Casse. Concerning a characterisation of Buekenhout-Metz unitals. J. Geom. 52 (1-2) (1995), 159-167.

[14] L. Storme, J.A. Thas and S.K.J. Vereecke. New upper bounds for the sizes of caps in finite projective spaces. J. Geom. 73 (2002), 176-193. 